\title{
Assessment of performance metrics for fusion network
}

\author{
Rohan Gupta ${ }^{1}$, Gurpreet Singh'2, Amanpreet Kaur ${ }^{3, *}$ \\ ${ }^{1}$ Dept. of Electronics and Communication Engineering,SJPMLIET, \\ Radaur, Haryana, India \\ ${ }^{2}$ Dept. of Computer Science Engineering, Punjab Institute of Technology, \\ Rajpura,MRSPTU, Bathinda, Punjab, India \\ ${ }^{3}$ University Institute of Engineering, $C U$, \\ Mohali, Punjab, India \\ *Corresponding Author:aman_preet_k@yahoo.co.in
}

\begin{abstract}
The arrangement which does not necessitate any infrastructure for doing discussion among nodes is called MANET. The direction-finding technique, a mixture of ACO (ant colony optimization) and particle swarm optimization (PSO) technique, is designed in the paper. The designed technique is judged against several alternatives of particle swarm optimization technique and ant colony optimization technique, i.e.,EDNR+PSO, AntChain Protocol, Improvised Ant Colony Routing (IACR), and ANTALG. Open-source simulator NS 2.3 is employed for carrying out the simulation. From the outcome, it has been verified that the designed technique is finest in contrast to other alternatives of PSO and ACO.Routing overheads of the designed algorithm are reduced in contrast to other methods.
\end{abstract}

Keywords: ACO; ANTALG; IACR; mobile ad-hoc network; PSO; QOS; routing protocols.

\section{Introduction}

The system which swaps the data with nodes and to achieve this action does not necessitate infrastructure is recognized as MANET. Effect of changingamount of nodes in MANET is inspected on diverse quality of service metrics,namely average delay, throughput, andpacket delivery ratio(Batth, $\mathrm{K}$ \& Singh, R., 2017). Three stages considered in the ant colony optimization are hunting a path, sustaining a route, and restoring the route (Guptav., 2018). Few shortcomings of ACO are overcome by hybrid ant colony optimization, which uses different transition and pheromone updating rules to attain improved solutions(Sedighpour et al.,2014). There are numerous uses where the mobile ad-hoc network is utilized in dispensing data with the aid of portable tools are military area, sensor networks, and commercial division (Gupta et al., 2017). The Ants favor a route of superior probability because this is reliant on the pheromone rate. Via this way, the extra quantity of pheromone shall be capable of capturing the focus of subsequent ants leading to productive response on productive paths and unnecessary on other lanes(Singh et al., 2016). The groups which function in the deficiency of central agent are termed as a mobile ad-hoc network. Locate the superior lane between sender and target is one of the chief issues in MANET(Kaur et al., 2016).

The ants capable of establishing the smallest route among the initial and target node are called blind ants ( $\mathrm{Li}$ et al., 2008). In MANET, all in-between nodes act as a router for information broadcasting. Diverse routing algorithms are employed to crack this problem. MANET sorts its routing algorithms into three types:- 


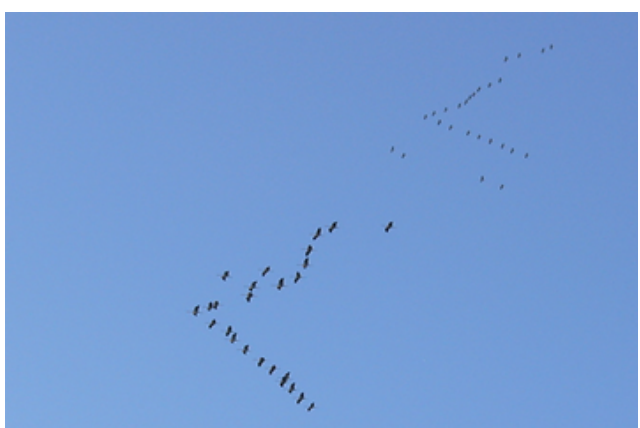

Fig.1.Flocking of Birds.

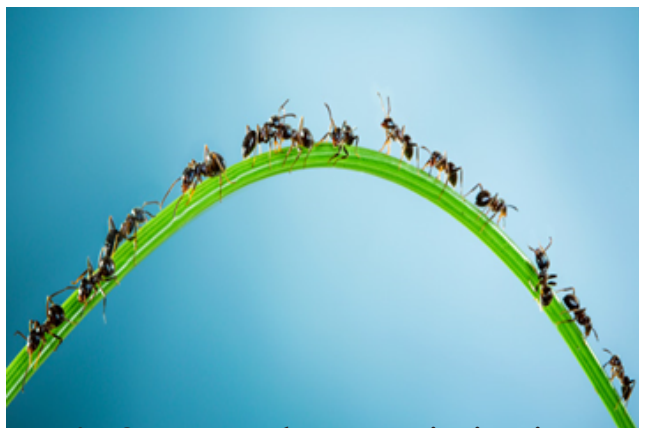

Fig.2. Ant Colony Optimization.

(i) Proactive (ii) Reactive (iii) Hybrid Protocols(Jha et al., 2015) (Bhatia et al., 2016).

Routing data in the Proactive algorithm is occasionally broadcast throughout the system. Diverse routing algorithms like OLSR, DSDV, etc., are a few examples of proactive algorithms. Few examples of reactive algorithms are DSR, AODV, WRP, $\mathrm{ABR}$, etc. A hybrid algorithm is a fusion of reactive and proactive techniques. These routing algorithms permit fast convergence. ZHLS and ZRP are examples of hybrid algorithms. Scientists are employing numerous algorithms to lessen the point of congestion(Batla et al., 2014) by use of a fusion. ACO attempts to crack trouble by associating a set of single agents referred to as artificial ants. ACO routing techniques create optimum lanes to the target using different artificial agents which converse not directly with one another by stigmergy(Socha, K.;Dorigo, M., 2008).

\section{Related work}

The wireless sensor systems in which nodes can be revitalized. Lane has been constructed on the principle of ant colony optimization technique. For creating the lane, the nodes which can be energized are employed, and for attaining the finest lane, the ant colony optimization algorithm is used(Huang et al., 2016).In ANTALG algorithm, during the route revival period the overheads will be diminished through the choice of starting and target nodes which are carried out arbitrarily. Pheromones are renewed globally for augmenting the probability of picking the optimized lane (Singh et al., 2014).E-DYMO in which ant agents have been employed for building traffic. It fuses ant colony optimization technique and DYMO for creating the path and locating the smallest path. It has better performance parameters than DYMO; however,the limitation exhibited by this research paper is larger routing overheads(Gupta et al.2014). A proficient ant colony optimization technique with the initiation of orientation factor is designed. Through the Orientation factor, the packet moves in the exact path, and chances of being tracked in the loop are diminished. Thus shortest lane, lesser routing overheads, and improved throughput are attained compared to the AODV routing algorithm(Kumar, P \& Prasad V. V., 2015). Routing technique TA-NARP makes use of the ant colony optimization technique for investigating the smallest lane among initial and target nodes and enhancing the lifetime of the system. This technique attains the least delay, enhanced throughput, and better packet delivery ratio(Mahale, $\mathrm{R} \&$ Chavan, S.,2014).ACO-DARP routing algorithm has the uniqueness of the AOMDV routing technique. It communicates in several phases. In the first part, ants are created, and in the second part, the ants have to pick the job from the existing list based on the pheromone. After that,pheromones have to be accumulated on the boundaries that ants have stayed. Finally, daemon action is carried out, and pheromone evaporation is done to avoid saturation and premature convergence(G.Sathishkumar, K. Vignesh, 2015). 
In the case of ACO, the antseventually speak by putting pheromones on the lane from the initial node to the target node. (Kaur et al., 2016). ). The author projected ACO-based multipath routing technique for uninterrupted conversation during the lane breaks(Kaur,A.;Dhaka, V. S.;Singh, G. (2016). The system topology plays an important role as the investigating space is defined through it(Duan et al., 2010). The author has proved that more favorable results are achieved through genetic algorithms as compared to PSO while analyzing a model of oil reservoir(Karimiet al., 2019). The author has conversed AACO routing technique which calculates the information about the finest path(Yu et al.,2017).To minimize energy consumption, the author proposed the SABAPSO technique(O.M, O.;Ayobami T, O.;B.A, O.;Adebimpe, E.;Bolanle, W. et al., 2020). To accomplish multipath transmission of information, MGWO-DSR has been initiated by the author(Ghaleb, S.; Vasanthi, V., 2020).

\section{Proposed work}

In a system topology, the ACO technique is suitable for hunting the path.PSO is mainly suitable for solving optimization difficulties because of its capability to handle population multiplicity. The designed technique uses ACO to determine the viable paths for particular system topology from starting node to destination node. The lanes are determined on the criterion of the node's leftover energy and bandwidth. Once the viable lanes are found out depending upon the pheromone concentration, the viable routes create a key PSO population. For particular initial nodedestination nodes, the most favorable lanes are predicted based on bandwidth and delay from the prime population. These lanes are recognized on the origin of speed, fitness function, and location update. The global best and particle best are considered for each element on the origin of energy and delay. The iterations are replicated till we appear the supreme set of element. Now the weaker outcome to the trouble is discarded, and only the finest solutions are retained, which essential situation for multipath routing.

\section{A. Design of ACO Algorithm}

- Imagine that the initial node has data to send out to the final node in which each connected node has a table holding information concerning the route.

- The data regarding the neighboring nodes are congregated by hello messages.

- The information accrued by forwarding ant comprises the existing potential of each connection and delay at each node etc.

- The backward ant will follow the analogous lane, which has been pursued by forwarding ant in a contradictory way.

- lane is followed on the origin of bandwidth, delay, and hops to appear at the initiating node.

- In case the probability of the resolute lane is superior in comparison to the requirement, then the lane is chosen, or else it is discarded.

$$
\Omega_{i j d}=\frac{\rho_{i j d}+\theta \Gamma_{i j}}{\theta\left|N_{i}\right|+(1-\theta)}(3.1)
$$

- Where $\mathrm{i}$ is the current node, $\mathrm{d}$ is the destination node, $\mathrm{j}$ is the chosen next node, $\rho$ is pheromone value, $\theta$ is the heuristic function. $\mathrm{Ni}$ represents the number of neighbors for node i. एis a heuristic value that is further dependent on the length of the queue over the link.

$$
\Gamma_{i j}=1-\left(e_{i j} / \sum_{i=1}^{|N i|} e_{i j}\right)
$$

Equation (3.3) explains the concept of pheromone update.

$$
\tau_{i j}=(1-\rho) \tau_{i j} \pm \rho \Delta \tau_{i j}(3.3)
$$

Mathematical Equations of the proposed 
algorithm are elaborated using equations number 3.1, 3.2, and 3.3.

The lane having superior probability is elected as the premium lane, and convey of data will take place by this lane.

\section{B. Design of PSO Algorithm}

- Each lane is considered a particle. To every particle, prime position and velocity are fixed. All particles have anindividual premium position, and there is a most favorable location between each and all of the particles.

- Fitness Function is utilized to compute how optimum is a place of an ith particle in significance to the selected objective.

- The next part is to decide on Pbest and Gbest.Every particle is linked with a preliminary position and velocity. Further, every particle is having its best position as $\mathrm{P}$ _best and the finest of all particles are encoded as $\mathrm{G}$ best

- Then the velocity is verified by deducting Gbest and Post.

- To realize the competent lane, carry out the grouping of nodes in the lane and path velocity. Packets are launched from the lane in which the node has the least quantity of cost.

\section{Simulation Environment}

Simulation has been done using NS-2 with topology usagebecause it is a well-recognized simulator and is open-source. The reason for doing a simulation on the technique is to test the features of services metrics. 100 nodes are used to accomplish the conversion.

Table 1. Parameters and associated values.

\begin{tabular}{||ll||}
\hline Parameter & Value \\
Protocols Studied & GPSR \\
Simulator & $\mathrm{N}-2$ \\
transmission range & $100 \mathrm{~m}, 250 \mathrm{~m}$ \\
Simulation Area & $1000 * 1000$ \\
& Random \\
Node Movement Model & waypoint \\
Data Payload & $1460 \mathrm{es} /$ packet \\
Simulation time & 250 seconds \\
Traffic type & CBR \\
& \\
\hline
\end{tabular}

Packet dimension reserved was equivalent to 512 Byte, and traffic type is Constant Bit Rate(CBR).

Table2. Comparison of different parameters.

\begin{tabular}{||llll||}
\hline \hline Algorithm & $\begin{array}{l}\text { Delivery } \\
\text { Ratio }\end{array}$ & Throughput & $\begin{array}{l}\text { Routing } \\
\text { overhead }\end{array}$ \\
Ant Chain & 85.63 & 81914 & 7.820 \\
IACR & 91.26 & 86662 & 6.354 \\
ANTALG & 78.66 & 87662 & 5.629 \\
EDNR+PSO & 99 & 89600 & 8.1 \\
Proposed & 99.153 & 90616 & 4.3 \\
\hline \hline
\end{tabular}

Two Ray Ground is used in simulation, which is one of the modes of Propagation. There are a variety of antennas like horn antenna, omnidirectional, yagi-uda antenna, and turnstile antenna. Among these, the antenna frequently in use is Omnidirectional. The Omnidirectional antennahas the feature that it transmits the waves in all ways. At $250 \mathrm{sec}$, the simulation will begin. The topology measurement, i.e., $1000 \times 1000$ array, is taken. Full duplex communication will be created among the nodes. Parameters and associated values are illustrated in Table 1. The broadcasting and reception happen concurrently in both directions in the fullduplex communication

\section{Metrics employed}

To test the competence of the designed technique, a variety of parameters have been judged.

\section{A. Throughput}

Quantity of digital information per unit time broadcast via a link that can be logical or physical. It is calculated in bits/second.

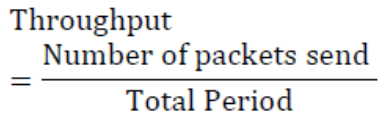

\section{B. Packet delivery ratio}

Estimation of one-hundredth of packets broadcast efficiently to the target node.

\footnotetext{
Delivery Ratio

(packets dispatched - damaged packets) $\times 100$ Packets dispatched
} 


\section{Routing Overhead}

Routing overhead for the routing technique means a division of the number of control packets to the number of messages conveyed.

$$
\begin{aligned}
& \text { overhead } \\
& =\frac{\text { Amount of Control Packets }}{\text { Amount of messages delivered }}
\end{aligned}
$$

\section{Results and discussion}

The assessment of the designed hybrid technique with dissimilar techniques of PSO and ACO in terms of a variety of quality of services matrices has been made. Different comments have been accomplished while doing the simulation. Comparison of different parameters is illustrated in Table 2.The proposed technique has been compared with Ant chain, IACR, ANTALG, EDNR+PSO. These Protocols have been taken for the comparison purpose because these are different variants of ant colony optimization (ACO) and particle swarm optimization (PSO).The paper has designed the hybrid approach, which is based on both the techniques of ACO and PSO. That is why we have used these algorithms forcomparison purposes.

The throughput of the hybrid algorithm is enhanced compared to other variants, whereas the packet delivery ratio has been augmented by just about $2 \%$. Routing overhead is decreased to around half in comparison to other variants. A plot of PDRVs simulation time is revealed in Figure 3.PDR of the ANTALG is least between all the algorithms.PDR of the Ant chain is too less, but it is elevated than ANTALG.IACR and EDNR+PSO have acceptable values of PDR, but the designhas the utmost value. From the plot, it has been depicted that the PDR of the designed algorithm is augmented by just about $2 \%$. Figure 4 reveals throughput concerning simulation time. The throughput of the Ant chain is the least compared to EDNR+PSO, IACR, ANTALG, and the designed algorithm. The throughput of the designed algorithm is high in comparison to other optimizationalgorithms. Figure 5 reveals the routing overhead concerning simulation time. EDNR+PSO, PSO variants, has maximum routing overheads compared to alternative ant colony optimization.

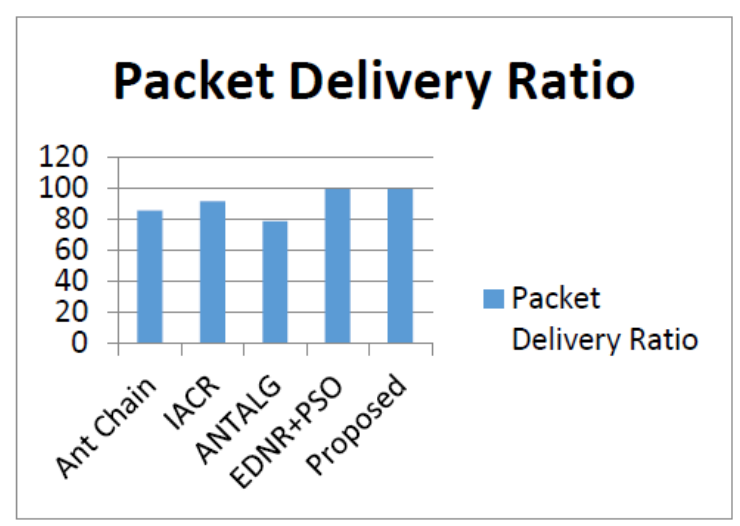

Fig.3. Delivery Ratio Vs. Simulation Time.

The routing overhead of IACR is too elevated, but it is fewer than EDNR+PSO and Ant chain. The routing overhead of the design is diminished to almost half.Thus, we can say that by hybridizing these approaches, the results achieved are better than when results are computed only by applying the ACO algorithm or PSO algorithm individually.

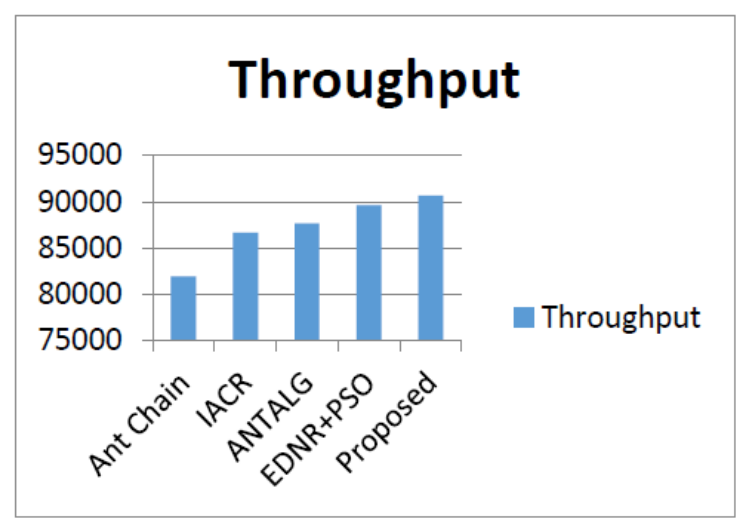

Fig.4.Throughput Vs. Simulation Time.

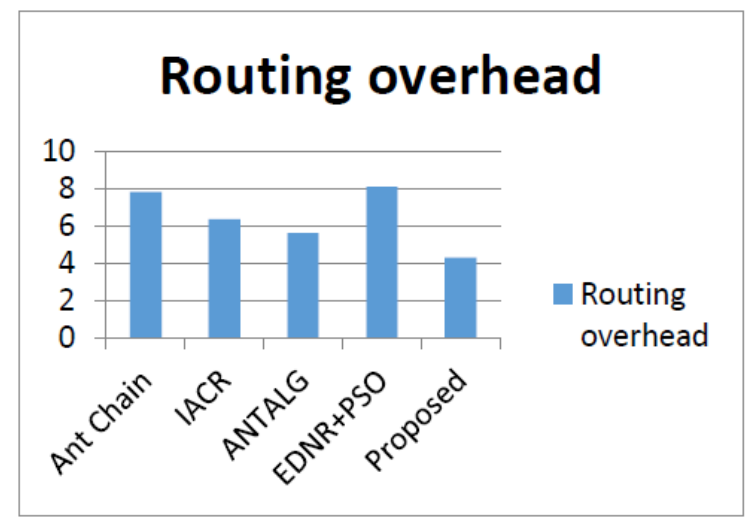

Fig.5. Routing Overhead Vs. Simulation Time. 


\section{Conclusion}

In this research paper,an evaluation of the quality of service metrics for fusion, the network has been carried out. The performance of the fusion system is judged against several variants of ACO like AntChain Protocol, Improvised Ant Colony Routing (IACR), ANTALG, and $\mathrm{EDNR}+\mathrm{PSO}$, which is one of the variants of PSO. It has been confirmed through the outcome that the designed hybrid technique is a supreme algorithm as compared to other optimization techniques in relevance to throughput, routing overhead, and packet delivery ratio.Hybridization of $\mathrm{ACO}$ and PSO algorithm is taken in this research paper as ACO algorithm is appropriate for finding the path, and PSO is particularly appropriate for resolving optimization troubles because of its ability to manage population multiplicity. Throughput is high in contrast to other optimization algorithms, whereas the packet delivery ratio has been enlarged by just about $2 \%$. Routing overhead is decreased to about half in comparison to other optimization techniques.

\section{Future Scope}

The designed algorithm can incorporate the energy model, which is one of the future works. Quality metrics taken in this research paper are packet delivery ratio,throughput, and routing overhead. However, few other parameters can be taken to judge the performance of a hybrid system. The military sector, commercial sector, home system, data networks, and sensor networks are few applications of this technique.

\section{References}

Batla, N.; Kaur, A.; Singh, G. (2014). Congestion Control Techniques in TCP: A Critique. Proceedings of 3rd National Conf. of Advances and Research in Technology (ART-2014), 45.1-45.5.
Bhatia, D.; Sharma, D. (2016). A Comparative Analysis of Proactive, Reactive and Hybrid Routing Protocols over open Source Network. Journal of Applied Engineering Research, 11: 3885-3896.

Duan, Q.; Liao, T. W. (2010). Improved ant colony optimization algorithms for determining project critical paths. Journal of Automation in Construction, 19, 676-693.

G. Sathishkumar; K. Vignesh. (2015). Ant Colony Optimization Based Delay Aware Routing Protocol (ACO-DARP) For Wireless Sensor Network. Journal of Research in Applied Science \& Engineering Technology, 3: 396-402.

Ghaleb, S.; Vasanthi, V. (2020). Energy Efficient Multipath Routing Using MultiObjective Grey Wolf Optimizer based Dynamic Source Routing Algorithm for MANET, International Journal of Advanced Science and Technology, 29: 6096 - 6117.

Gupta, A.K.; Sadawarti, H., \& Verma, A.K.(2014). Performance Enhancement of DYMO Routing Protocol with Ant Colony Optimization. Journal of Electrical and Electronics Engineering 2: 188-194.

Gupta, R.; Singh, G.; Kaur, A.; Singh, A. (2018). Fitness function-based particle swarm optimization algorithm for mobile ad-hoc networks. Journal of Engineering and Technology,7(3.1): 31-33.

Gupta, R.; Singh, H.\& Singh, G. (2017). Performance Evaluation of Routing Protocols for Mobile Ad-hoc Networks. Journal of Science and Technology, 10: 1-6.

Huang, P.; Kang, Z.; Liu, C.\& Lin, F. (2016). ACO-Based Path Planning Scheme in RWSN Proceedings of the 10th IEEE Conference on Software, Knowledge, Information Management \& Applications, 237-242. 
Jha, R.; Kharga, P. (2015). A Comparative Performance Analysis of Routing Protocols in MANET using NS3 Simulator. Journal of Computer Network \& Info. Security, 4: 6268.

Karimi, M.; Mortazavi, A.; Ahmadi, M. (2019). Applying an optimized low-risk model for fast history matching in a giant oil reservoir. Kuwait Journal of Science, 46 (1): 84-89.

Kaur, A.; Dhaka, V. S.; Singh, G. (2016). Casting multipath behavior into OANTALG to improve QoS. Proceedings of the conference on Computing for Sustainable Global Development, 2076-2081.

Kaur, A.; Dhaka, V. S.; Singh, G.(2016). ACO Agent-Based Routing in AOMDV Environment. Int. Conf. on Advances in Engineering \& Technology-2016, MATEC Web of Conferences, 57:1-8.

Kumar, P.\&Prasad V. V. (2015). Efficient Ant Colony Optimization (ACO) based Routing Algorithm for MANETs. Global Journal of Computer Science and Technology,15(3).

Mahale, R.\& Chavan,S.(2014). Throughput Aware ACO Based Routing Protocol for Wireless Sensor Network. Proceedings of the IEEE Conference Publications, 234-238.

O. M, O.; Ayobami T, O.; B.A, O.; Adebimpe, E.; Bolanle, W. et al.(2020). Power Aware And Secured Routing Protocol In Mobile Ad-Hoc Network: A Survey, International Journal of Advanced Research in Engineering and Technology, 11: 706-717.

Sedighpour, M.; Ahmadi, V.; Yousefikhoshbakht, M.; Didehvar, F., and Rahmati, F. (2014). Solving the open vehicle routing problem by hybrid ant colony optimization. Kuwait Journal of Science, 41(3):139-162.
Singh, A.; Dhaka, V. S.; Singh, G. (2016). Comparative Analysis of Dynamic Path Maintenance Routing Protocols for Mobile Ad-Hoc Networks. Journal of Science and Technology, 9:1-6.

Socha, K.;Dorigo, M.(2008). Ant colony optimization for continuous domains. European Journal of Operation Research Elsevier, 185:1155-1173.

Yu, K.; Lee, M.; Chi, S. (2017). Dynamic Path Planning Based on Adaptable Ant Colony Optimization algorithm. Proceedings of the Sixth International Conference on Future Generation Communication Technologies,1- 7 .

Submitted:

$16 / 05 / 2020$

Revised: $\quad 16 / 09 / 2020$

Accepted: $\quad 20 / 09 / 2020$

DOI: $\quad 10.48129 /$ kjs.v48i3.9751 